\title{
Corilagin suppresses cholangiocarcinoma progression through Notch signaling pathway in vitro and in vivo
}

\author{
YUE GU ${ }^{1,2}$, LINFENG XIAO $^{1}$, YANLIN MING ${ }^{3}$, ZHIZHONG ZHENG ${ }^{3}$ and WENGANG LI ${ }^{1,4}$ \\ ${ }^{1}$ Medical College of Xiamen University, Xiamen; ${ }^{2}$ Fujian Institute of Subtropical Botany, Xiamen; \\ ${ }^{3}$ The Research and Development Center for Medicine Plants and Plant Drugs, Xiamen Overseas \\ Chinese Subtropical Plant Introduction Garden, Xiamen; ${ }^{4}$ Department of Hepatobiliary Pancreas \\ and Vessel Surgery, Chenggong Hospital of Xiamen University, Xiamen, Fujian, P.R. China
}

Received December 24, 2015; Accepted February 4, 2016

DOI: $10.3892 /$ ijo.2016.3413

\begin{abstract}
Corilagin is a natural plant polyphenol tannic acid with antitumor, anti-inflammatory, and anti-oxidative properties. However, the mechanisms of its actions are largely unknown. Our group reported that corilagin could induce cell inhibition in human breast cancer cell line MCF-7 and human liver hepatocellular carcinoma cell lines HepG2. We report here that corilagin inhibits cholangiocarcinoma (CCA) development through regulating Notch signaling pathway. We found that, in vitro, corilagin inhibited CCA cell proliferation, migration and invasion, promoted CCA cell apoptosis, and inhibited Notch1 and Notch signaling pathway protein expression. Co-immunoprecipitation was used to establish Notch intracellular domain (NICD) interaction with MAML1 and P300 in CCA. Importantly, corilagin reduced Hes1 mRNA level through inhibiting Hesl promoter activity. In nude mice, corilagin inhibited CCA growth and repressed the expression of Notch1 and mTOR. These results indicate that corilagin may control CCA cell growth by downregulating the expression of Notch1. Therefore, our findings suggest that corilagin may have the potential to become a new therapeutic drug for human CCA.
\end{abstract}

\section{Introduction}

Cholangiocarcinoma is a common malignancy of the biliary tract. It has a high mortality rate, and is difficult to diagnose (1). The morbidity and mortality of intrahepatic

Correspondence to: Professor Wengang Li, Department of Hepatobiliary Pancreas and Vessel Surgery, Chenggong Hospital of Xiamen University, 94-96 Wenyuan Road, Xiamen City, Fujian 361000, P.R. China

E-mail: lwg11861@163.com

Abbreviations: CCA, cholangiocarcinoma; NICD, Notch intracellular domain; $\mathrm{CSL}, \mathrm{CBF} 1 / \mathrm{Su}(\mathrm{H}) / \mathrm{Lag}-1$

Key words: corilagin, cholangiocarcinoma, proliferation, migration, invasion, apoptosis, Notch signaling pathway cholangiccarcinoma in the world are on the rise (2). The cause for this increase could be related to an interplay between genetic factors and environmental triggers (3). Chemotherapy can lead to drug resistance and thus worse prognosis (4). Surgical resection is the only chance for cure. The 5-year survival rate is $<5 \%$ making it one of the leading causes of death (5). The conventional chemotherapy drugs used are Oxaliplatin and Mitomycin, which are prone to resistance by cancer cells thus compromising the efficacy of the drugs $(6,7)$. Therefore, it is necessary to search for new drugs that are more effective and with less side effects.

Corilagin ( $\beta$-D-glucopyranose,cyclic3,6-[(1R)-4,4',5,5', 6,6'-hexahydroxy(1,1'-biphenyl)-2,2'-dicarboxylate]1-(3,4,5trihydroxybenzoate) is a polyphenol tannic acid from plant extracts. It has both anti-inflammatory and anti-oxidant properties, and is commonly used for treating bacterial and viral infections, and diabetes $(8,9)$. The molecular formula of corilagin is $\mathrm{C}_{27} \mathrm{H}_{22} \mathrm{O}_{18}$, and its molecular weight is 634.45 (structure is shown in Fig. 1). Corilagin inhibited the growth of E.coli and C. albicans by disrupting their membrane permeability but not acting on membrane integrity (10). Corilagin could attenuate TBHP-induced oxidative stress in microcells, and its protective effect may be due to its antioxidant and anti-apoptotic properties, which made corilagin a potential candidate for the treatment of oxidative stress-induced neurodegenerative diseases (11). Corilagin significantly reduced the production of pro-inflammatory cytokines and mediators such as TNF- $\alpha$, IL-1 $\beta$, IL-6 and COX-2 (12).

In the past few years, research on corilagin was focused on its use as an antiviral, hypolipemic, hypotensive and anticoagulation agent (13). However, there is scarce data on the effect of corilagin on cancer. A study shows that corilagin arrests SMMC7721 cells at the G2/M phase by downregulating p-Akt and cyclin B1/Cdc2 and upregulating p-p53 and p21Cip1 (14). Another study (14) of corilagin in human gastric cancer demonstrated that corilagin nanoparticle induced cell apoptosis via the mitochondrial pathway. These observations suggest that corilagin possesses a potential anticancer effect. However, the role of corilagin in cholangiccarcinoma remains largely unknown. In our preliminary studies, we demonstrated that CCA cell proliferation, migration, invasion and apoptosis were affected by corilagin treatment. 
The Notch signaling pathway is an evolutionarily conserved pathway which plays an important role in cell fate determination, proliferation, differentiation an survival $(16,17)$. The Notch system in vertebrates comprises four receptors: Notch1, Notch2, Notch3 and Notch4 and at least five ligands from the families Delta and JAG/Serrate (DSL): JAG1, JAG2, Delta-like (D1l)-1, Dll-3 and Dll-4 (18,19). Membrane localization of Notch requires S1 cleavage of precursor of the Notch receptor. This event occurs in the Golgi network by the action of a furinlike convertase.

Activation of Notch consists of two consecutive cleavages of the transmembrane receptor upon the binding of a Notch ligand, which triggers S2 cleavage. S2 cleavage releases the Notch extracellular domain (NECD) from the heterodimer and creates a membrane tethered Notch extracellular truncation (NEXT), which becomes a substrate for $\gamma$-secretase. S3 is cleaved by $\gamma$-secretase at sites 3 and 4 . This last cleavage occurs on the plasma membrane and/or in endosome. The new mobile cytoplasmic subunit [Notch intracellular domain (NICD)] is translocated to the nucleus, where it interacts with members of the DNA-binding protein, recombination signal binding protein for immunoglobulin kappa J (RBP-JK) or CBF1/Su(H)/Lag1 (CSL) family of transcription factors $(20,21)$. Now we known that there are several mechanisms of corilagin in a tumor, such as MAPK, NF- $\kappa$ B and TGF- $\beta 1$ signaling (22). However the involvement of corilagin in the regulation of Notch pathway remains unknown. In this study, we investigated the effect of corilagin regulated tumor development and occurrence through Notch signaling pathway in vitro and in vivo.

\section{Materials and methods}

Cancer cell culture. The human cancer cells QBC9939, MZ-Cha-1 were obtained from the American Type of Culture Collection (ATCC, Rockville, MD, USA). They were separately cultured in 100-mm culture dish (Corning Glass Works, Corning, NY, USA) using RPMI-1640 and DMEM medium (Gibco, Grand Island, NY, USA) with 5\% fetal bovine serum (Gibco), $100 \mu \mathrm{g} / \mathrm{ml}$ streptomycin and $100 \mathrm{U} / \mathrm{ml}$ penicillin at $37^{\circ} \mathrm{C}$ in $5 \% \mathrm{CO}_{2}$ humidified incubator.

Cell proliferation assay. 3-(4,5-Dimethylthiazol-2-yl)-2,5diphenyl-2H-tetrazolium bromide (MTT, Amresco, Solon, OH, USA) was used to detect the effect of corilagin on the prolifera-

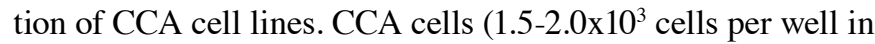
$100 \mu 1$ medium) were seeded in 96-well plates and incubated with corilagin $(0,10,20,30,40,50,60,70,80,90 \mu \mathrm{mol} / \mathrm{l})$ starting the following day and continuing for $48 \mathrm{~h}$. Then a solution of $5 \mathrm{mg} / \mathrm{ml}$ MTT was dissolved in PBS and $20 \mu \mathrm{l}$ of MTT solution was added from step one to each well containing cells. The plate was incubated at $37^{\circ} \mathrm{C}$ for $4 \mathrm{~h}$. Media was remove and $150 \mu \mathrm{l}$ DMSO (Sigma-Aldrich, St. Louis, MO, USA) was added to wells with vibration to dissolve crystals, and the plate was incubated for $5 \mathrm{~min}$. The absorbance at wavelength $570 \mathrm{~nm}$ was measured using a plate reader.

Wound-healing assay. CCA cells ( $2 \times 10^{5}$ cells/well) were plated in a 6-well plate, and a scratch created using a sterile $10 \mu \mathrm{l}$ pipette tip when they were confluent, then washed twice to remove non-adherent cells. Cells were cultured in normal medium and treated with corilagin at $40 \mu \mathrm{M}$ for $48 \mathrm{~h}$. Photomicrographs were captured at $48 \mathrm{~h}$ after wounding using a microscope.

Invasion assay. The invasion assay was performed in 24-well transwell plates (Millipore, Billerica, MA, USA) with uncoated, $8-\mathrm{mm}$ pore, polycarbonate membranes. In brief, serum-starved cells were harvested and re-suspended at a concentration of $2 \times 10^{5}$ cells $/ \mathrm{ml}$ in serum-free medium, and $200 \mu \mathrm{l}$ was added to the upper chamber. After treatment with corilagin for $48 \mathrm{~h}$, CCA cells that had not invaded were discarded using a cotton swab. Cells that had invaded the lower compartment were stained with $0.1 \%$ crystal violet. The number of cells on the lower side of the membrane was quantified by average cell counts from six random fields in each well, under a microscope at x200 magnification, and images were captured.

Cell cycle analysis. CCA cells were seeded in 60-mm plates $\left(1-2 \times 10^{5}\right.$ cells/plate) and incubated with corilagin (20-40 $\mu \mathrm{mol} / \mathrm{l})$ or DMSO as a control the next day. Cells were harvested after $24 \mathrm{~h}$ of treatment, washed with PBS, fixed with ice-cold $70 \%$ ethanol, and incubated at $4{ }^{\circ} \mathrm{C}$ overnight. The cells were then washed with PBS and treated with $10 \mu \mathrm{g} / \mathrm{ml}$ RNase (Amresco) for $30 \mathrm{~min}$ at room temperature. Cell nuclei were stained with $50 \mu \mathrm{g} / \mathrm{ml}$ propidium iodide (PI, Sigma) for $15 \mathrm{~min}$ at room temperature in the dark and detected by flow cytometry (BD Biosciences, Franklin Lakes, NJ, USA). The cell cycle distribution was analyzed using ModFit3.0 software.

Apoptosis analysis. CCA cells were seeded in a $60-\mathrm{mm}$ dish $\left(1-2 \times 10^{5}\right.$ cells/dish) and incubated with corilagin (20-40 $\mu \mathrm{mol} / \mathrm{l})$ or DMSO as a control. The cells were harvested after $24 \mathrm{~h}$ of incubation, washed with PBS, and stained with Annexin V-fluorescein and PI according to the manufacturer's instructions for the Annexin V-FLUOS Staining kit (Roche, Mannheim, Germany). The stained cells were analyzed by flow cytometry.

Real-time PCR analysis. Total RNA was extracted by the guanidinium thiocyanate-phenol-chloroform method, purified, and subjected to reverse transcription with random hexanucleotide primers (Takara, Foster City, CA, USA). The resulting cDNA was then subjected to real-time PCR analysis with SYBR Green PCR master mix (Applied Biosystems, Foster City, CA, USA) and $200 \mathrm{nM}$ gene-specific primers. Assays were performed in triplicate with a StepOnePlus realtime PCR system (Applied Biosystems). The amplification protocol comprised initial incubation at $60^{\circ} \mathrm{C}$ for $30 \mathrm{sec}$ and at $95^{\circ} \mathrm{C}$ for $3 \mathrm{sec}$, followed by 40 cycles. The sequences of the various primers (sense and antisense, respectively) are listed below: 5'-AGCTGCCTTTCATTTAGCACTCTAC-3' and 5'-TTAAGACTTTCCAGGGTATATCCAGTC-3' for Cyclin A, 5'-TACCTATGCTGGTGCCAGTG-3' and 5'-CACATCC AGATGTTTCCATTG-3' for Cyclin B, 5'-AGAGGCGGA GGAGAACAAAC-3' and 5'-TGAGGCGGTAGTAGGAC AGG-3' for Cyclin D, 5'-GGATTGTGGCCTTCTTTGAGT-3' and 5'-TCAAACAGAGGTCGCATGCT-3' for Bcl-2, 5'-GCC GAGGCTTGAGGTATATT-3' and 5'-TCC TTCTTCAGAG GCAGCAT-3' for Caspase 3, 5'-TATCATGGAGAAGAG 


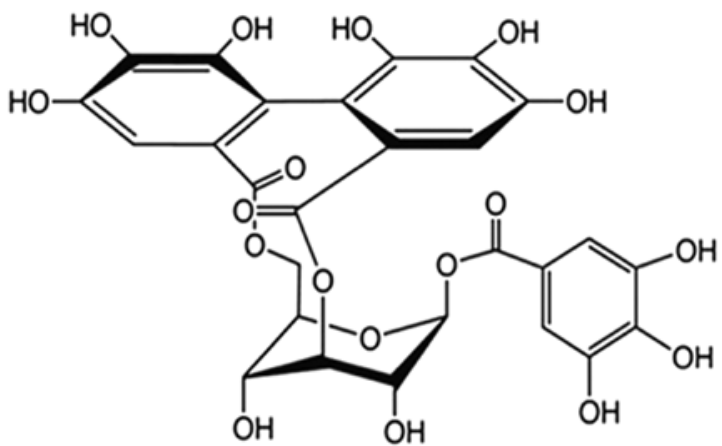

Figure 1. The chemical structure of corilagin $\left(\mathrm{C}_{27} \mathrm{H}_{22} \mathrm{O}_{18}\right)$. The molecular weight is 634.45 .

GCGAAGG-3' and 5'-TTCTCTAGCTTGGAATGCCGG-3' for Hes1, 5'-CTACCCAAAAGTAATCATCTTAAGTG-3' and 5'-CCCAACCATGACAAGATTTTCC-3' for Fbw7 exon8, 5'-TTTCTGTTTCTCCCTCTG-3' and 5'-GAGCATTTAAG GGAGAGATAAGAG-3' for Fbw7 exon9, 5'-TCTTCCAGCC TTCCTTCCT-3' and 5'-AGCACTGTGTTGGCGTACAG-3' for $\beta$-actin.

Western blot analysis. The CCA cells were harvested and lysed in RIPA buffer (1\% Triton, $0.1 \%$ SDS, $0.5 \%$ deoxycholate, $1 \mathrm{mM}$ EDTA, $20 \mathrm{mM}$ Tris (pH 7.4), $150 \mathrm{mM} \mathrm{NaCl}$, $10 \mathrm{mM} \mathrm{NaF}, 1 \mathrm{mM} \mathrm{Na} \mathrm{VO}_{4}, 0.1 \mathrm{mM}$ phenylmethylsulfonyl fluoride). Then, cell lysates were cleared by centrifugation and a BCA assay (Pierce, Rockford, IL, USA) was used to detect the concentration of protein in the supernatants using a Varioskan multimode microplate spectrophotometer (Thermo Fisher Scientific, Waltham, MA, USA). Protein was prepared for the same concentration. The prepared protein was separated by SDS-PAGE and transferred onto nitrocellulose (NC) membranes. The nitrocellulose membranes were blocked with $10 \%$ non-fat milk in PBS at $37^{\circ} \mathrm{C}$ for $1 \mathrm{~h}$ and incubated with primary antibodies (Cell Signaling Technology, Danvers, MA, USA) overnight at $4^{\circ} \mathrm{C}$. After washing three times for $10 \mathrm{~min}$ each in PBST, membranes were incubated with HRP-conjugated secondary antibody for $1 \mathrm{~h}$ at $37^{\circ} \mathrm{C}$. Chemiluminescence detection was performed using SuperSignal WestDura Extended Duration Substrate (Pierce), and bands were visualized using the Automatic digital Imaging Analysis System (Tanoo-5500, Shanghai, China).

Animals and treatment. Eight weeks old athymic nude mice, weighing approximately 15-20 g, were purchased from the animal unit of Xiamen University and maintained in a sterile facility, in accordance with the institutional guidelines on animal care, with the required consistent temperature and relative humidity. All the procedures were approved by the Animal Research Ethics Committee. Sixteen athymic nude mice were injected subcutaneously with the human CCA cells. They were housed in sterile conditions. Tumor size was measured by an electronic calliper daily. When tumor size reached a mean volume of $200 \mathrm{~mm}^{3}$, where tumor volume was calculated by the formula (length $\mathrm{x}$ width $\mathrm{x}$ width)/2, they were randomly divided into four groups. Corilagin at a concentration of $20 \mathrm{mg} / \mathrm{kg}$ were administrated intraperitoneally for a
A

QBC939

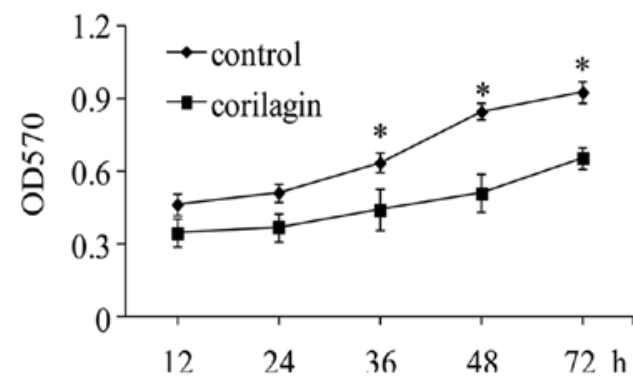

B

MZ-Cha-1

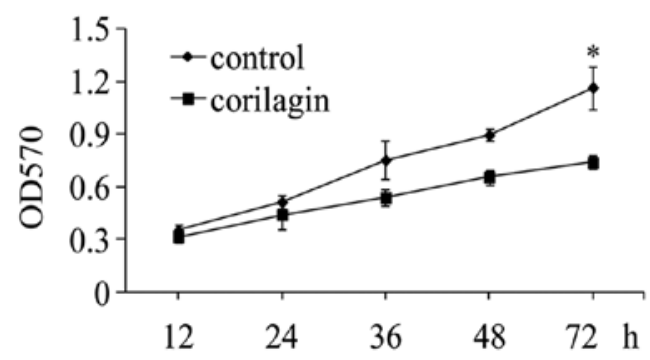

Figure 2. Corilagin suppresses the proliferation of cholangiocarcinoma (CCA) cell lines. Evaluation of the cell proliferation curve by MTT assays. The CCA cell lines QBC939 (A) and MZ-Cha-1 (B) were treated with $30 \mu \mathrm{mol} / 1$ corilagin for the indicated times. The data were obtained from three experiments and represent means $\pm \mathrm{SD}$. " $\mathrm{P}<0.01$ versus control cells.

continuous period of 3 days starting from day 1 to day 30 . Control group received equal volume of vehicle intraperitoneally. Each group consisted of 4 mice. On day 30, all the mice were sacrificed and tumors were collected for further investigation.

Statistical analysis. The data was analyzed from at least three independent experiments. The statistical significance of differences between two groups was evaluated using Student's t-test. SPSS 13.0 software (SPSS Inc., Chicago, IL, USA) was used to carry out all the statistical analyses. P-values were two-sided, and $v$ P-value $<0.05$ was considered to indicate significance. The data are presented as the mean \pm SD.

\section{Results}

Corilagin effectively suppresses CCA cell proliferation. To investigate the effect of corilagin on CCA cell proliferation, we first determined the $50 \%$ cell inhibitory concentration $\left(\mathrm{IC}_{50}\right)$ of corilagin. QBC939 and MZ-Cha-1 cells were treated in vitro with increasing concentrations of corilagin for $48 \mathrm{~h}$ and then analyzed by the MTT assay. The means \pm standard errors of $\mathrm{IC}_{50}$ were $39.73 \pm 1.28 \mu \mathrm{mol} / \mathrm{l}$ for QBC939 cells and $36.88 \pm 1.32 \mu \mathrm{mol} / 1$ for MZ-Cha-1 cells, respectively. Then we treated the cell lines with $30 \mu \mathrm{mol} / 1$ of corilagin for the indicated times (Fig. 2A and B). The result showed that corilagin could efficiently suppress CCA cell proliferation at 24 and $72 \mathrm{~h}$.

Corilagin decreases CCA cell cycle progression. As corilagin inhibited cell proliferation, cell cycle analysis was performed 
A

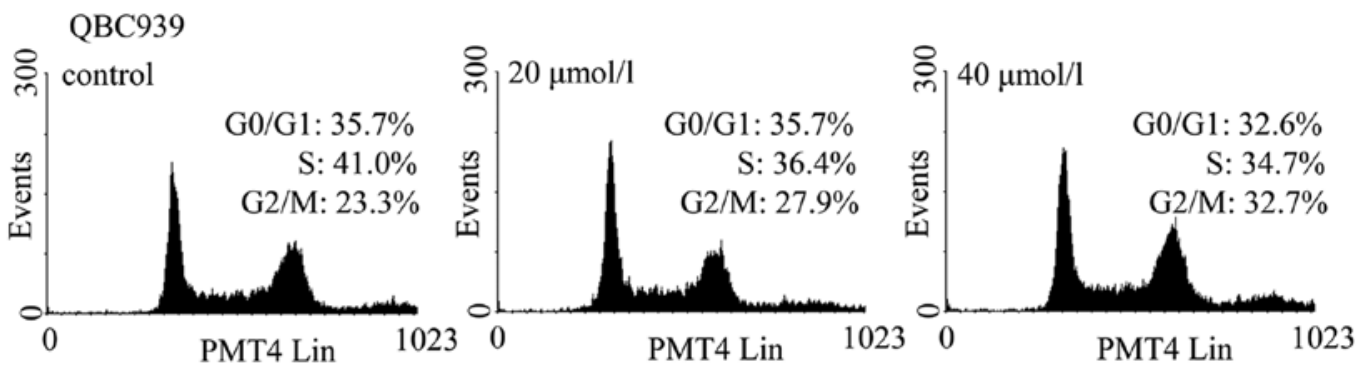

$\mathrm{B}$
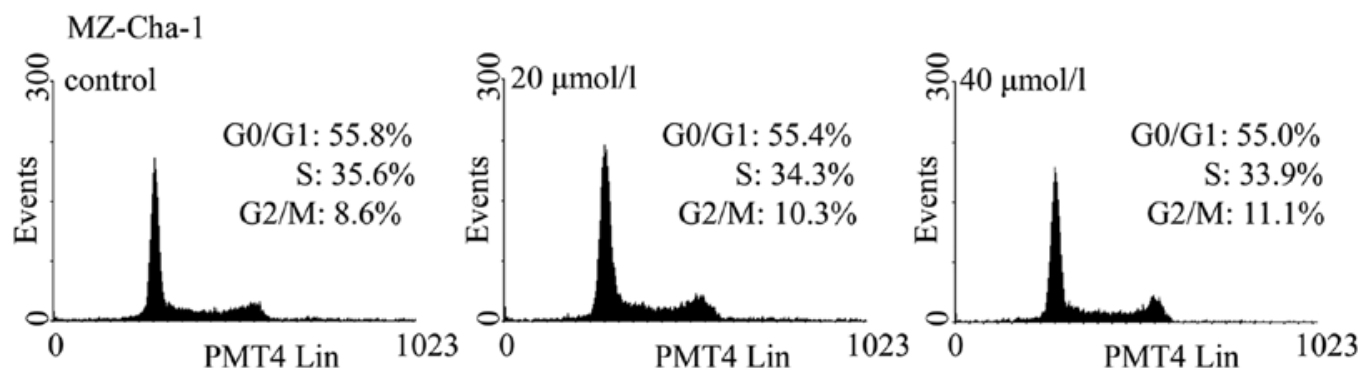

Figure 3. Corilagin induces cell cycle arrest (G2/M phase) in cholangiocarcinoma cells. QBC939 and MZ-Cha-1 cells were treated with corilagin at increasing concentrations for $24 \mathrm{~h}$ and analyzed for cell cycle arrest by flow cytometry. The effects of different treatments on the cell cycle distributions of QBC939 (A) and MZ-Cha-1 (B) cells.

to examine whether corilagin could induce cell cycle arrest. Two CCA cell lines were treated with corilagin at increasing concentrations for $24 \mathrm{~h}$ and then stained with PI. Flow cytometric analysis showed that the proportions of CCA cells treated with corilagin at G2/M phase was increased compared with control cells, and this was associated with a decrease in proportion of cells at S phase (Fig. 3). In QBC939 cells, the proportion of cells in the $\mathrm{G} 2 / \mathrm{M}$ phase increased from $23.3 \%$ to $27.9 \%$ and $32.7 \%$ with 20 and $40 \mu \mathrm{M}$ corilagin, respectively (Fig. 3A). Subsequently, inhibition of the G1 phase and S phase was observed. Corilagin has the same effect in MZ-Cha-1 cells, but with less potency (Fig. 3B). These results suggest that corilagin regulates cell cycle by arresting CCA cells in the $\mathrm{G} 2 / \mathrm{M}$ phase, and inhibiting cell growth.

To explore the mechanism underlying the changes in cell cycle progression, several cell cycle-related genes were examined by real-time PCR. The mRNA levels were compared between control cells and corilagin treated cells. The result showed that there was no significant changes in the expression of cyclin A, cyclin B and cyclin D. The exact mechanism of corilagin regulation of cell cycle progression needs to be explored in future studies.

Corilagin inhibits migration and invasion of CCA cells. The processes of migration and invasion are integral to embryonic development and the functioning of adult organisms. Deregulation of these processes contributes to the development of tumor and ultimately metastasis $(23,24)$. We tested whether corilagin could inhibit the migration and invasion of CCA cells. We performed monolayer wound healing assay to evaluate the effect of corilagin on migration of QBC939 cells. A scratch was created with a pipette tip when the cells were confluent, and treated with corilagin at $40 \mu \mathrm{M}$ for $48 \mathrm{~h}$. The result indicated that corilagin significantly decreased cell migration (Fig. 4A). For transwell invasion assay, QBC939 and MZ-Cha-1 cells were treated with corilagin at indicated concentrations for $12 \mathrm{~h}$. The number of cells that had invaded was less after corilagin treatment compared with control in QBC939 cells (Fig. 4B). Similar result was observed in MZ-Cha-1 cells (Fig. 4D). Together, these results suggest that corilagin could significantly decrease the migration and invasion of CCA cells.

Corilagin induces CCA cell apoptosis. The flow cytometry analysis of Annexin V-Fluos and PI staining revealed the quantity of viable (Annexin $\mathrm{V}^{-} / \mathrm{PI}^{-}$), early apoptotic (Annexin $\mathrm{V}^{+} / \mathrm{PI}^{-}$), late apoptotic (Annexin $\mathrm{V}^{+} / \mathrm{PI}^{+}$) and necrotic (Annexin $\mathrm{V}^{-} / \mathrm{PI}^{+}$) cells. To assess the antitumor effects of corilagin on CAA cells, we investigated the impact of corilagin on apoptosis. QBC939 and MZ-Cha-1 cells were incubated with increasing concentrations of corilagin. As shown in Fig. 5A and B, for both QBC939 and MZ-Cha-1 cells, the percentages of early apoptotic cells increased after treatment with corilagin. After $40 \mu \mathrm{M}$ corilagin treatment in QBC939 cells, the early apoptotic population was elevated from 6.9 to approximately $14.0 \%$, and the late apoptotic population was elevated from $2.9 \%$ to approximately $4.6 \%$, whereas, in MZ-Cha-1 cells the early apoptotic population was $19.4 \%$ and the late apoptotic was $10.4 \%$, and compared with control 15.4 and $5.4 \%$, respectively. To further investigate the apoptotic molecular mechanism, we detected the apoptosis-related gene bcl-2 and caspase 3 using real-time PCR in CCA cells. We examined the genes at early $(12 \mathrm{~h})$ and late $(24 \mathrm{~h})$ stages of corilagin treatment. As shown in Fig. 5C, the expression of bcl-2 mRNA was significantly decreased at 12, $24 \mathrm{~h}$ after corilagin treatment. While caspase 3 mRNA was strongly increased at 12, $24 \mathrm{~h}$ (Fig. 5D). These results indicated that bcl-2 and caspase 3 were involved in the corilagin-induced CCA cell apoptotic process. 
B

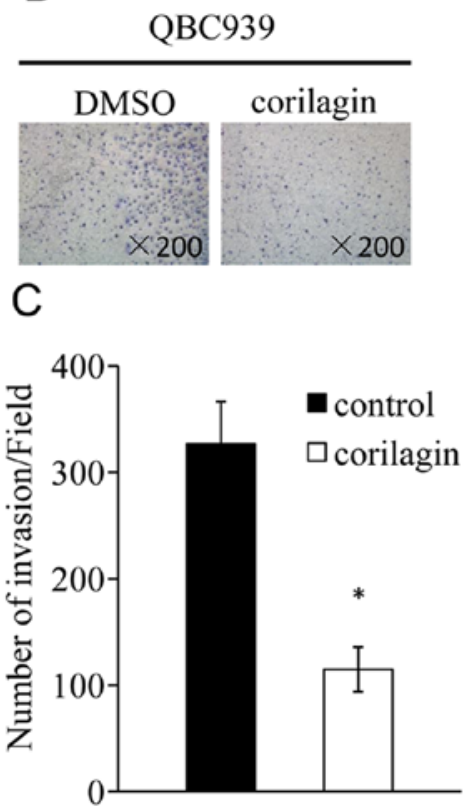

D

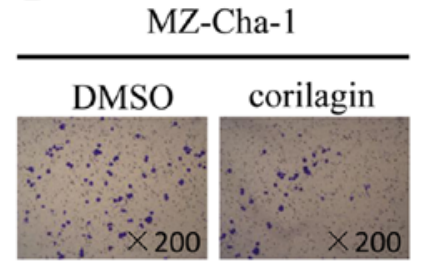

E

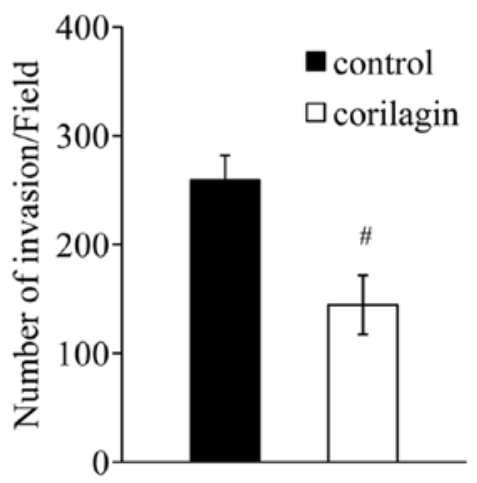

Figure 4. Corilagin inhibits migration and invasion of CCA cells. CCA cells were analysed for wound healing and cell invasion following treatment with corilagin. (A) Light microscopic images of QBC939 cells scratched with a pipette tip when they were confluent (upper panel), and after treatment with $40 \mu \mathrm{mol} / 1$ corilagin for $48 \mathrm{~h}$ (lower panel). (B and D) Light microscopic images of CCA cells, which have migrated through pores of the polycarbonate filters to the lower membrane surface after treatment with $40 \mu \mathrm{mol} / 1$ corilagin for $12 \mathrm{~h}$. Cells were stained with crystal violet. (C and E) Cell migration (cells/field) of CCA cells, as assessed by the transwell migration assay. The results shown are representative of three independent experiments and represent means \pm SD. ${ }^{*} \mathrm{P}<0.01,{ }^{\#} \mathrm{P}<0.05$ versus control cells.

Corilagin effectively suppresses Notch-mTOR pathway. NotchmTOR pathway plays an important role in the development and cell fate determination (25). To determine whether corilagin was involved in Notch-mTOR pathway, we firstly identified the protein expression patterns of Notch-mTOR signaling in CCA cells. Western blot analyses showed that corilagin had significant effects on Notch1 at 12, 24, $48 \mathrm{~h}$ after corilagin treatment; moreover, corilagin strongly inhibited the expression of mTOR at $48 \mathrm{~h}$ (Fig. 6A). In addition, we investigated the expression of $\mathrm{p}$-Akt and p-Erk1/2, the direct target gene of Notch that function as an important downstream effector in Notch-mTOR pathway. As shown in Fig. 6A, the expression of p-Akt and p-Erk1/2 was significantly reduced at $48 \mathrm{~h}$ after corilagin treatment.

Notch receptors are synthesized in the endoplasmic reticulum as an inactive single peptide precursor, which is proteolytically cleaved by a furin-like convertase in the transGolgi network before it reaches the plasma membrane (26). The third cleavage production, Notch intracellular domain (NICD), binding to ubiquitous transcription factor CSL, mastermindlike protein (MAML1) and P300 has been reported (27). In order to determine the interactions between NICD, MAML1 and P300, we performed a co-immunoprecripitation assay in CCA cells co-transfected with HA-NICD, Flag-MAML1, and MYC-NICD, HA-P300, respectively. The result showed that Flag-MAML1 was co-immunoprecipitated with HA-NICD (Fig. 6B), and HA-P300 was co-immunoprecipitated with MYC-NICD (Fig. 6C), suggesting that NICD interacted with MAML1 and P300 in CCA cells. To further confirm the effect of corilagin on Notch signaling, 293T cells transfected with NICD were treated with or without corilagin. As shown in Fig. 6D, NICD enhanced the phosphorylation of AKT. In contrast to NICD, corilagin decreased the phosphorylation of AKT, and counteracted the phosphorylation of AKT induced by NICD (Fig. 6D, lane 4). It is noteworthy that NICD inhibited the expression of Notch1 (Fig. 6D, lane 2), and this might be due to the negative feedback regulation (28). Secretase inhibitor DAPT, which affects Notch signal pathway, is used to treat Notch-mediated cancer such an breast cancer (29).

So far there is scarce data reported on DAPT used as a therapy in CCA. In order to confirm the role of corilagin in Notch active CCA cells, we assessed the effect of DAPT in CCA cells treated with corilagin and DAPT. As shown in Fig. 6E, DAPT inhibited the expression of Notch1, p-AKT and p-Erk1/2, while corilagin reduced expression of these proteins, which was consistent with previous studies. Corilagin and DAPT co-procession had a more significant inhibitory effect on Notch1, p-AKT, and p-Erk1/2. To determine whether corilagin was involved in the regulation of the transcriptional activity of Notch1 target genes, a reporter assay was conducted in QBC939 cells using the luciferase reporter gene. In mammals, the best-characterized Notch target gene belongs to the Hes family (30).

To confirm that corilagin affected the CCA cells through the Notch-mTOR pathway, we investigated the mRNA level of Hes1, which is one of hairy/enhancer of split (HES) family proteins. We found that the mRNA level of Hes1 was significantly decreased after corilagin treatment compared with the control group (Fig. 6F). Furthermore, we examined the transcriptional activity of Hes1 promoter after corilagin treatment. As shown in Fig. 6G, corilagin reduced the transcriptional activity of Hes1 promoter. These results 
A

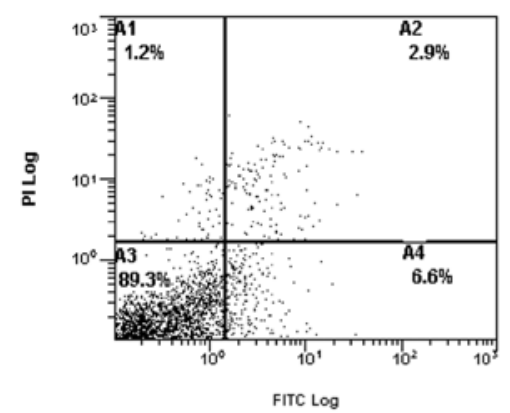

control

B

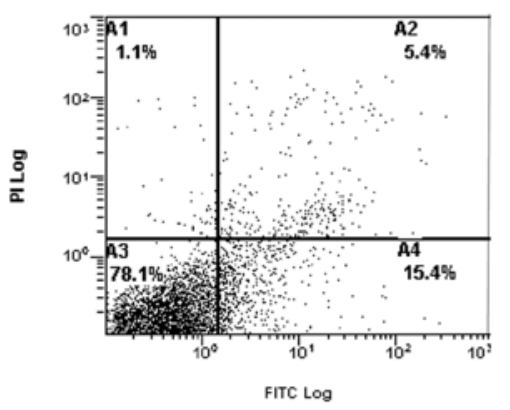

control

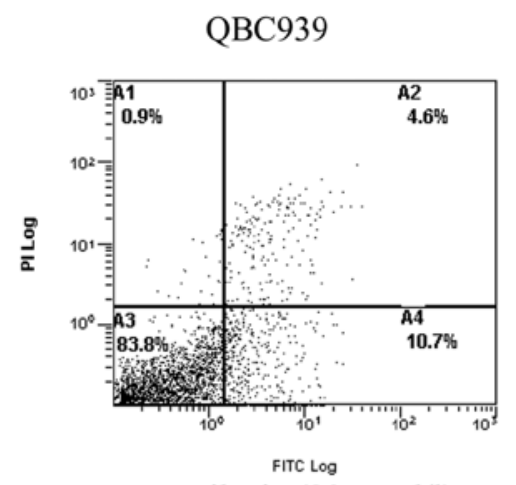

corilagin $(20 \mu \mathrm{mol} / \mathrm{l})$

MZ-Cha-1

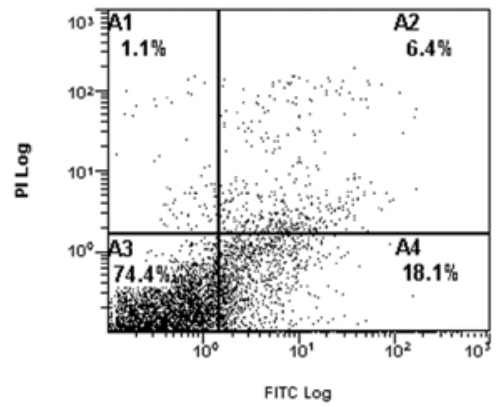

corilagin $(20 \mu \mathrm{mol} / \mathrm{l})$

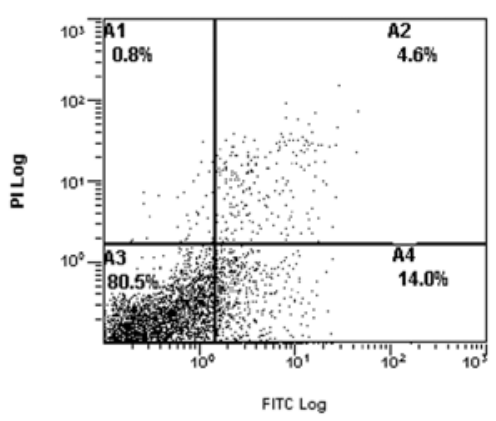

corilagin $(40 \mu \mathrm{mol} / \mathrm{l})$

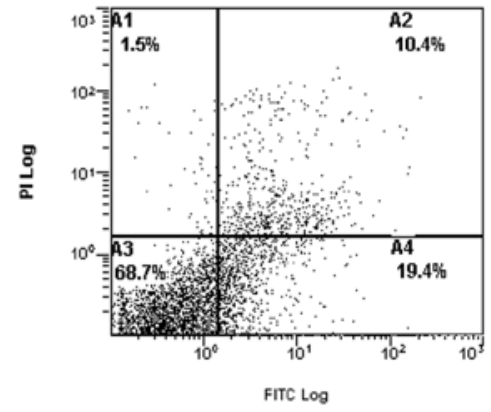

corilagin $(40 \mu \mathrm{mol} / \mathrm{l})$
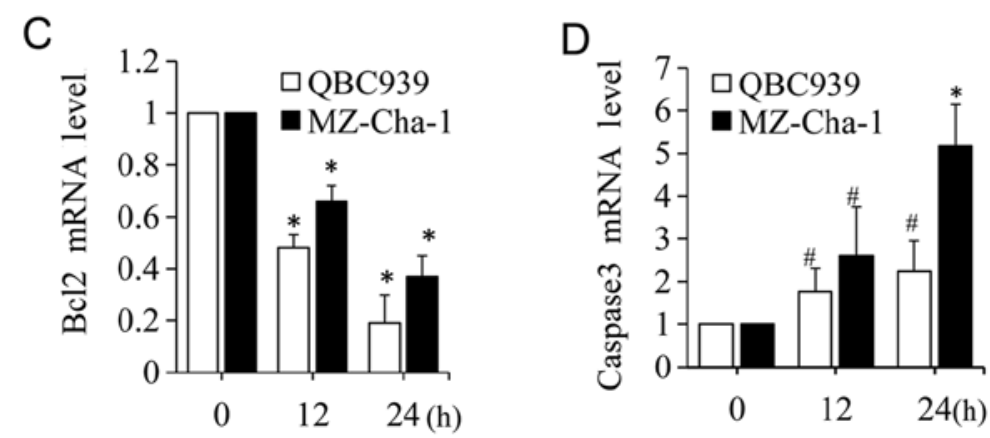

Figure 5. Corilagin induces CCA cell apoptosis. (A and B) Two CCA cell lines (QBC939 and MZ-Cha-1) were treated with corilagin at increasing concentrations for $24 \mathrm{~h}$ and analyzed for apoptosis by flow cytometry. (C and D) QBC939 and MZ-Cha-1 cells were harvested and RNA was isolated from each group. SYBR green real-time PCR reactions were performed to detect the alterations of bcl-2 (C) and caspase 3 (D) gene expression treated with corilagin for $24 \mathrm{~h}$. Each column represents the mean of three independent experiments. ${ }^{*} \mathrm{P}<0.01,{ }^{*} \mathrm{P}<0.05$.

suggested that corilagin could reduce the Hes1 mRNA level through inhibiting its promoter activity. All the results above suggested that corilagin might affect the notch signal pathway by restraining the active cleavage NICD, thereby affecting the transcriptional activity upon binding of NICD and ubiquitous transcription factor CSL which regulated the expression of target gene Hes1, and eventually altered the expression of downstream proteins such as p-AKT, p-Erk1/2. Thus, corilagin regulated Notch signal pathway in CCA cells.

Corilagin inhibits tumor growth progression in vivo. To investigate the effect of corilagin on the growth of CCA xenograft tumors in nude mice, we injected either vehicle or corilagin intraperitoneally. Tumor volume was quantified using electronic calliper. Corilagin treated CCA tumors grew much slower than control tumors. At the end of study (30 days), corilagin-treated mice exhibited markedly reduced tumors as compared with control mice (Fig. 7A and B). To further study the role of corilagin in CCA, we investigated the expression of Notch1 and mTOR in vivo. Proteins were extracted from xenograft tumors in a tumor-bearing nude mouse model. As shown in Fig. 7C and D, the expression of Notch1 and mTOR were significantly reduced in tumors from corilagin treated group compared with the control group, which was consistent with the in vitro experiments described above. Therefore, corilagin regulated the Notch-mTOR pathway in vivo as well as in vitro.

\section{Discussion}

Corilagin is a gallotannin identified in several plants and is the major active component of Phyllanthus niruri L. Phyllanthus niruri $\mathrm{L}$. is a well-known medicinal plant that has shown hepatoprotective, antiviral, antibacterial, analgesic, antispasmodic and anti-diabetic effects; however, there are few studies describing its antitumor activity. Herein we investigated the effect of corilagin on CCA and found its distinct 
A
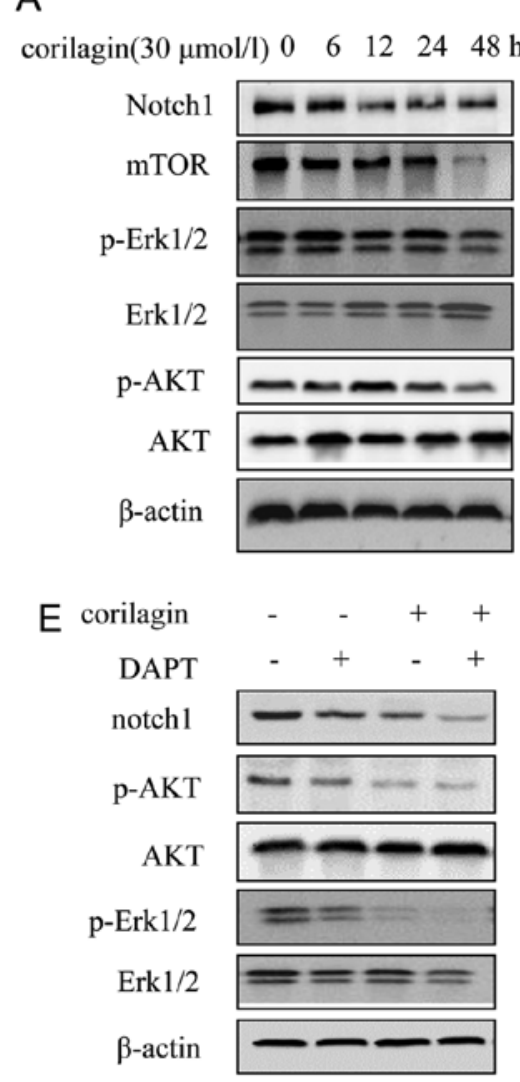

B

Input IgG HA

corilagin

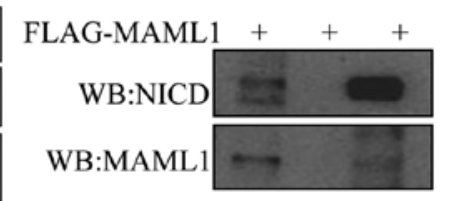

NICD

NICD

notch1

$\mathrm{p}-\mathrm{AKT}$

C

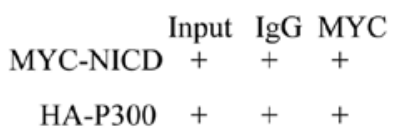

AKT

$\beta$-actin

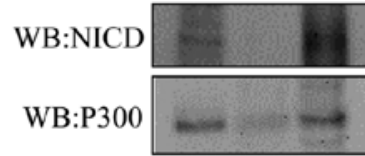

G
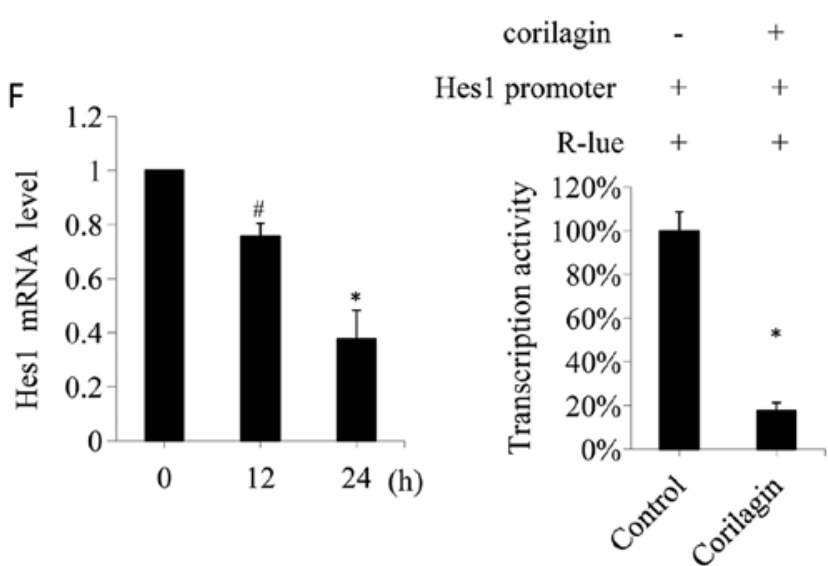

Figure 6. Corilagin suppress Notch-mTOR pathway and inhibits Notch1 transcriptional activity in CCA cells. (A) QBC939 cells were treated with $30 \mu$ mol/1 corilagin for different times, the expressions of notch1, mTOR, pERK1/2 and pAKT were detected by western blot analyses. (B) QBC939 cells were cotransfected with HA-NICD and FLAG-MAML1, and cell lysates were IP for HA, the immunoprecipitates were anlyzed by western blot for NICD and MAML1. (C) QBC939 cells were co-transfected with MYC-NICD and HA-P300, and cell lysates were IP for MYC, the immunoprecipitates were analyzed by western blotting for NICD and P300. (D) QBC939 cells transfected with either control vector or NICD-expressing construct were treated with or without corilagin, and the expression of NICD, notch1, AKT and pAKT were detected by western blotting. (E) QBC939 cells were treated with corilagin $(30 \mu$ mol/1) alone or in combination with DAPT $(1 \mathrm{nmol} / \mathrm{l})$ for $24 \mathrm{~h}$, cell lysates were subjected to western blot analysis for the expression of notch1, pERK1/2 and pAKT. (F) QBC939 cells were treated with $30 \mu \mathrm{mol} / 1$ corilagin for indicated time and the mRNA level was detected by real-time PCR. (G) Corilagin increased the activities of HES1-promoter in QBC939 cells, reporter activities were measured and normalized. ${ }^{*} \mathrm{P}<0.01,{ }^{\#} \mathrm{P}<0.05$.

antitumor activity. First we examined the effect of corilagin on phenotype of CCA cell lines and found that corilagin was able to inhibit the proliferation and cell cycle, reduce migration and invasion, and promote apoptosis of CCA cells, which suggested that corilagin play an important role in the development of CCA. Corilagin inhibited CCA cell proliferation by inducing $\mathrm{G} 2 / \mathrm{M}$ phase arrest, but had no significant changes in $\mathrm{G} 2 / \mathrm{M}$ phase in MZ-Cha-1 cells after corilagin treatment. These results indicated that cell growth inhibition might be mainly through some other ways, such as promoting MZ-Cha-1 cells apoptosis and necrosis. According to the following results we confirmed that corilagin simultaneously promoted early and late apoptosis in CCA cells. Corilagin inhibited the mRNA level of bcl-2 and promoted caspase 3 associated with apoptotic gene detection.

According to our results, corilagin could restrain the expression of Notch1, which showed a time gradient decreases. We found corilagin not only reduced Hes1 promoter activity, but also inhibited the Hes1 mRNA level. Using co-immunoprecipitation method we found that the active form of Notch intracellular hydrolysis of NICD was able to form a complex with P300 and MAML1 which could combine with CSL to initiate transcription. Corilagin regulating Notch signal pathway possibly inhibited the expression of Notch1 thus reducing the NICD form. In the absence of NICD, CSL performed transcriptional suppression state which restrained the activity of PI3K/AKT expression. After addition of the Notch signal pathway inhibitor DAPT, we found that DAPT treatment alone could inhibit the protein expression of Notch1, p-AKT and p-Erk1/2. When corilagin was combined with DAPT, the inhibition became more enhanced. Furthermore, NICD plasmid was transfected in CCA cells, and p-AKT and p-Erk1/2 expression increased as compared to control group, which was a direct effect of NICD promotion on the expression of downstream target genes; whereas, exogenous NICD caused decreased expression of Notch1, which was a negative feedback inhibition mechanism that has been reported (31). These results suggested that corilagin effect the expression of downstream target protein Notch1, mTOR, $\mathrm{p}-\mathrm{AKT}$ and $\mathrm{p}-\mathrm{Erk} 1 / 2$ to regulate the occurrence and development of CCA through Notch signal pathway, as shown in Fig. 8. 
A
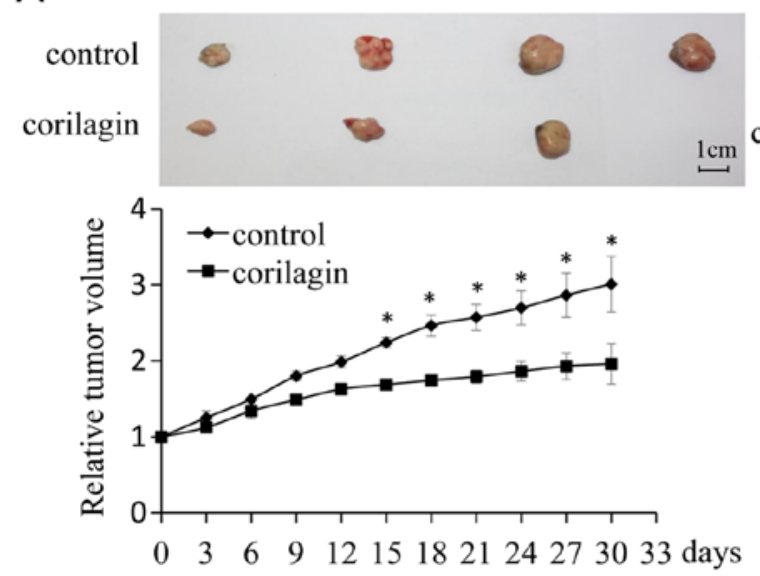

C

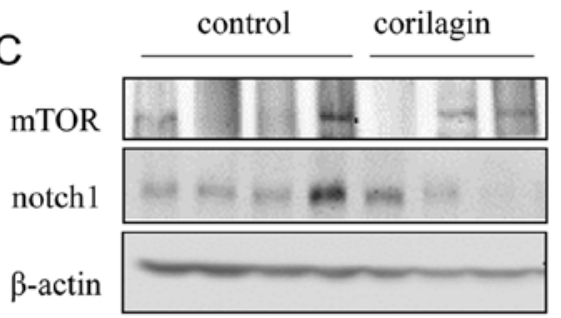

B
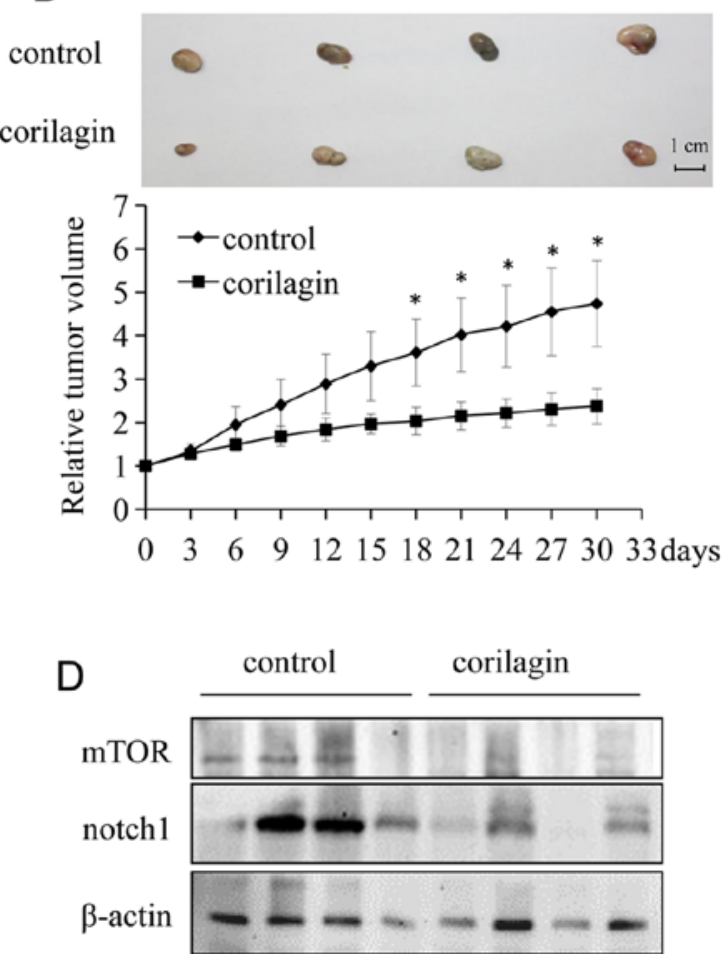

Figure 7. Corilagin inhibits CCA cell growth and downregulates Notch1 in vivo. (A) Corilagin decreased QBC939 cell growth in vivo. Upper panel, the image of tumors; lower panel, quantitative analysis of tumor weight. * $\mathrm{P}<0.05$. (B) Corilagin decreased MZ-Cha- 1 cell growth in vivo. Upper panel, the image of tumors; lower panel, quantitative analysis of tumor weight. ${ }^{*} \mathrm{P}<0.05$. (C) The protein levels of Notch1, mTOR in QBC939 tumors were detected by western blotting. (D) The protein levels of Notch1, mTOR in MZ-Cha-1 tumors were detected by western blotting.

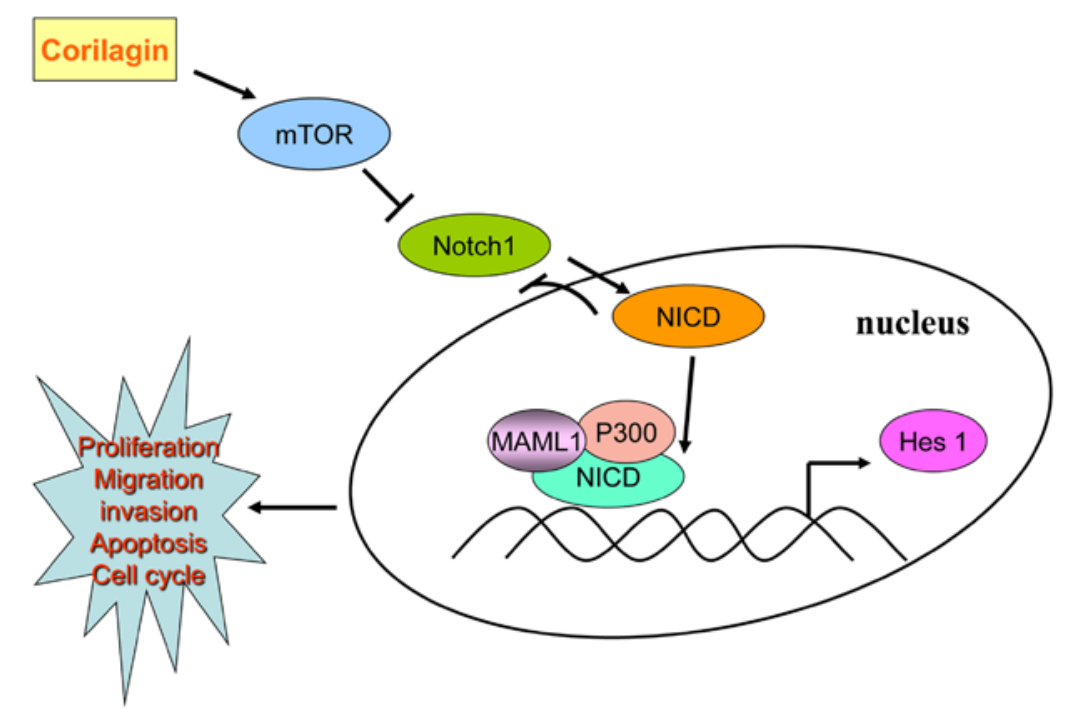

Figure 8. Molecular mechanisms by corilagin suppresses cholangiocarcinoma progression through Notch signaling pathway.

In consideration of the effect of corilagin on CCA cells in vitro, we further demonstrated that corilagin profoundly suppressed CCA growth and significantly inhibited Notch1 and mTOR protein expression in vivo. It is well established that, angiogenesis is an underlying promoter of tumor growth, invasion, and metastases (32). We transfected $293 \mathrm{~T}$ cells with NICD plasmid and found that NICD did not effectively restore corilagin reduced p-AKT protein level. This showed, corilagin could regulate other pathways besides NICD such as NF-kb (12). However which other pathways are involved in CCA treatment with corilagin still needs further exploration.

Current antitumor drugs have poor selectivity and high toxicity. Research on the action of natural product drugs promise to reveal the intricate interplay of biology, that will provide new opportunities for drug discovery. In our research, corilagin significantly inhibited CCA cell growth with lower 
cytotoxic effects on normal cells. To further investigate corilagin role in CCA, we confirmed Notch signal pathway involved in CCA occurrence and development treated with corilagin. corilagin enhanced the cytotoxic effect of CCA cells combined with common chemotherapeutic drugs. Corilagin, a monomer component of traditional Chinese medicine, is now pushed to the front, and could became a major herbal antitumor drug based on its safety and effective features. With the recent technological advances, a new golden age of nature products drug discovery is dawning (33). We consider that corilagin is a new promising candidate, as a potential antitumor drug for treating cancer.

\section{Acknowledgements}

This work was supported by funds from the National Natural Science Foundation of China (grant number: 81272246 to W.L.), the Xiamen Municipal Science and Technology Innovation Fund Project (grant number: 3502Z20124049 to W.L.). This work was also supported by grants from the Natural Science Foundation of China (grant number: 81274149 to Y.M.). We thank Professor C.D. Yu (Key Laboratory of Xiamen University) for providing a number of regents. We also thank Mr. D.Q.Zeng (School of Pharmacy, Xiamen University) for technical support in analysis.

\section{References}

1. Singal AK, Vauthey JN, Grady JJ and Stroehlein JR: Intrahepatic cholangiocarcinoma - frequency and demographic patterns: Thirty-year data from the M.D. Anderson Cancer Center. J Cancer Res Clin Oncol 137: 1071-1078, 2011.

2. McGlynn KA, Tarone RE and El-Serag HB: A comparison of trends in the incidence of hepatocellular carcinoma and intrahepatic cholangiocarcinoma in the United States. Cancer Epidemiol Biomarkers Prev 15: 1198-1203, 2006.

3. Al-Bahrani R, Abuetabh Y, Zeitouni N and Sergi C: Cholangiocarcinoma: Risk factors, environmental influences and oncogenesis. Ann Clin Lab Sci 43: 195-210, 2013.

4. Tepsiri N, Chaturat L, Sripa B, Namwat W, Wongkham S, Bhudhisawasdi V and Tassaneeyakul W: Drug sensitivity and drug resistance profiles of human intrahepatic cholangiocarcinoma cell lines. World J Gastroenterol 11: 2748-2753, 2005.

5. Shaib Y and El-Serag HB: The epidemiology of cholangiocarcinoma. Semin Liver Dis 24: 115-125, 2004.

6. Poggi G, Quaretti P, Minoia C, Palumbo I, Villani L, Amatu A, Teragni C, Scelsi M, Zappoli F and Bernardo G: Oxaliplatin-eluting microspheres for the treatment of intrahepatic cholangiocarcinoma: A case report. Anticancer Res 28: 2987-2990, 2008.

7. Shitara K, Ikami I, Munakata M, Muto O and Sakata Y: Hepatic arterial infusion of mitomycin $\mathrm{C}$ with degradable starch microspheres for unresectable intrahepatic cholangiocarcinoma. Clin Oncol (R Coll Radiol) 20: 241-246, 2008.

8. Yeo SG, Song JH, Hong EH, Lee BR, Kwon YS, Chang SY, Kim SH, Lee SW, Park JH and Ko HJ: Antiviral effects of Phyllanthus urinaria containing corilagin against human enterovirus 71 and Coxsackievirus A16 in vitro. Arch Pharm Res 38: 193-202, 2015.

9. Rangkadilok N, Sitthimonchai S, Worasuttayangkurn L, Mahidol C, Ruchirawat M and Satayavivad J: Evaluation of free radical scavenging and antityrosinase activities of standardized longan fruit extract. Food Chem Toxicol 45: 328-336, 2007.

10. Li N, Luo M, Fu YJ, Zu YG, Wang W, Zhang L, Yao LP, Zhao CJ and Sun Y: Effect of corilagin on membrane permeability of Escherichia coli, Staphylococcus aureus and Candida albicans. Phytother Res 27: 1517-1523, 2013.

11. Chen Y and Chen C: Corilagin prevents tert-butyl hydroperoxideinduced oxidative stress injury in cultured N9 murine microglia cells. Neurochem Int 59: 290-296, 2011.
12. Gambari R, Borgatti M, Lampronti I, Fabbri E, Brognara E, Bianchi N, Piccagli L, Yuen MC, Kan CW, Hau DK, et al: Corilagin is a potent inhibitor of NF-kappaB activity and downregulates TNF-alpha induced expression of IL-8 gene in cystic fibrosis IB3-1 cells. Int Immunopharmacol 13: 308-315, 2012.

13. Prasad KN, Yang B, Shi J, Yu C, Zhao M, Xue S and Jiang Y: Enhanced antioxidant and antityrosinase activities of longan fruit pericarp by ultra-high-pressure-assisted extraction. J Pharm Biomed Anal 51: 471-477, 2010.

14. Wang B: Corilagin nanoparticle-induced apoptosis in human gastric cancer SGC-7901 cells via the mitochondrial pathway. Acta Pharmacol Sin 34: 14-14, 2013

15. Ming Y, Zheng Z, Chen L, Zheng G, Liu S, Yu Y and Tong Q: Corilagin inhibits hepatocellular carcinoma cell proliferation by inducing G2/M phase arrest. Cell Biol Int 37: 1046-1054, 2013.

16. Ranganathan $P$, Weaver KL and Capobianco AJ: Notch signalling in solid tumours: A little bit of everything but not all the time. Nat Rev Cancer 11: 338-351, 2011.

17. Takebe N, Harris PJ, Warren RQ and Ivy SP: Targeting cancer stem cells by inhibiting Wnt, Notch, and Hedgehog pathways. Nat Rev Clin Oncol 8: 97-106, 2011.

18. Miele L, Golde T and Osborne B: Notch signaling in cancer. Curr Mol Med 6: 905-918, 2006.

19. Miele L, Miao H and Nickoloff BJ: NOTCH signaling as a novel cancer therapeutic target. Curr Cancer Drug Targets 6: 313-323, 2006.

20. Zhou W, Wang G and Guo S: Regulation of angiogenesis via Notch signaling in breast cancer and cancer stem cells. Biochim Biophys Acta 1836: 304-320, 2013.

21. Borggrefe $T$ and Oswald F: The Notch signaling pathway: Transcriptional regulation at Notch target genes. Cell Mol Life Sci 66: 1631-1646, 2009.

22. Wang Z, Guo QY, Zhang XJ, Li X, Li WT, Ma XT and Ma LJ: Corilagin attenuates aerosol bleomycin-induced experimental lung injury. Int J Mol Sci 15: 9762-9779, 2014.

23. Kimpton WG, McKenzie GA, Muller HK, Ruby JC and Poskitt DC: Lymphocyte migration during the development of regional lymph node anergy in experimental tumor growth. Cell Immunol 75: 13-21, 1983.

24. Takahashi S, Hasebe T, Oda T, Sasaki S, Kinoshita T, Konishi M, Ueda T, Ochiai T and Ochiai A: Extra-tumor perineural invasion predicts postoperative development of peritoneal dissemination in pancreatic ductal adenocarcinoma. Anticancer Res 21: 1407-1412, 2001.

25. El-Habr EA, Levidou G, Trigka EA, Sakalidou J, Piperi C, Chatziandreou I, Spyropoulou A, Soldatos R, Tomara G, Petraki K, et al: Complex interactions between the components of the PI3K/AKT/mTOR pathway, and with components of MAPK, JAK/STAT and Notch-1 pathways, indicate their involvement in meningioma development. Virchows Arch 465: 473-485, 2014.

26. Takebe N, Nguyen D and Yang SX: Targeting notch signaling pathway in cancer: Clinical development advances and challenges. Pharmacol Ther 141: 140-149, 2014.

27. Oswald F, Täuber B, Dobner T, Bourteele S, Kostezka U, Adler G, Liptay S and Schmid RM: p300 acts as a transcriptional coactivator for mammalian Notch-1. Mol Cell Biol 21: 7761-7774, 2001.

28. Stella MC, Trusolino L, Pennacchietti S and Comoglio PM: Negative feedback regulation of Met-dependent invasive growth by Notch. Mol Cell Biol 25: 3982-3996, 2005.

29. Zhao L, Ma Y, Gu F and Fu L: Inhibition of Notch1 increases paclitaxel sensitivity to human breast cancer. Chin Med J (Engl) 127: 442-447, 2014.

30. Kuang SQ, Fang Z, Zweidler-McKay PA, Yang H, Wei Y, Gonzalez-Cervantes EA, Boumber Y and Garcia-Manero G: Epigenetic inactivation of Notch-Hes pathway in human B-cell acute lymphoblastic leukemia. PLoS One 8: e61807, 2013.

31. Huppert SS, Jacobsen TL and Muskavitch MA: Feedback regulation is central to Delta-Notch signalling required for Drosophila wing vein morphogenesis. Development 124: 3283-3291, 1997.

32. Trédan O, Lacroix-Triki M, Guiu S, Mouret-Reynier MA, Barrière J, Bidard FC, Braccini AL, Mir O, Villanueva C and Barthélémy P: Angiogenesis and tumor microenvironment: Bevacizumab in the breast cancer model. Target Oncol 10: 189-198, 2015.

33. Shen B: A new gloden age of natural products drug discovery. Cell 163: 1297-1300, 2015. 